\title{
Time trend of asthma prevalence among school children in Taiwan: ISAAC phase I and III surveys
}

\author{
Lee Y-L, Hwang B-F, Lin Y-C, Guo YL and Taiwan ISAAC Study \\ Group. Time trend of asthma prevalence among school children in \\ Taiwan: ISAAC phase I and III surveys. \\ Pediatr Allergy Immunol 2007: 18: 188-195. \\ (C) 2007 The Authors \\ Journal compilation @ 2007 Blackwell Munksgaard
}

The standardized International Study of Asthma and Allergies in Childhood (ISAAC) is a valid tool in assessing prevalence of asthma indices. In order to determine the time trends of childhood asthma in Taiwan, we compared data from nationwide ISAAC surveys from a very large sample of Taiwanese 12- to 15-year-old school children, using ISAAC core written and video questionnaires. The number of participants was 44,104 in 1995-96 (phase I) and 11,048 in 2001 (phase III). We found a general tendency towards an increase in lifetime prevalence of physician-diagnosed asthma and asthma symptoms between two surveys, more marked for girls than for boys. Most of the 12-month prevalence of asthma symptoms decreased among boys but stabilized among girls. When comparing different severity levels, we also noted that the decreasing trends of current symptoms were more marked with regard to severe symptoms than mild symptoms in both sexes.

A combination of both improved awareness and management of asthma might in part explain this circumstance. Over the past decade in Taiwan, the lifetime prevalence of childhood asthma was increasing, more marked among girls; however, the 12-month prevalence of asthma symptoms was decreasing, mostly among boys. The exact reasons for such trends remain to be explored.

\begin{abstract}
Yung-Ling Lee ${ }^{1}$, Bing-Fang Hwang ${ }^{2}$, Ying-Chu Lin ${ }^{3}$, Yueliang Leon Guo and Taiwan ISAAC Study Group*

${ }^{1}$ Department of Occupational and Environmental Medicine, College of Medicine, National Cheng Kung University, Tainan, Taiwan, ${ }^{2}$ School and Graduate Institute of Occupational Safety and Health, College of Public Health, China Medical University, Taichung, Taiwan, ${ }^{3}$ College of Dental Medicine, Kaohsiung Medical University, Kaohsiung, Taiwan, ${ }^{4}$ Department of Environmental and Occupational Medicine, National Taiwan University (NTU) and NTU Hospital, Taipei, Taiwan
\end{abstract}

Key words: asthma; children; prevalence; trend; ISAAC; Taiwan

Yueliang Leon Guo, Department of Environmental and Occupational Medicine, College of Medicine, National Taiwan University, 1 Sec 1, Jen-Ai Road, Taipei 100, Taiwan

Tel.: +836 233228215

Fax: +836233228216

E-mail: leonguo@ha.mc.ntu.edu.tw

Accepted 26 October 2006
Asthma is the single most common chronic disorder in childhood (1), which has been reported as increasing around the world over the past

\footnotetext{
*Taiwan ISAAC Study Group includes the following investigators: Chien-Jen Chen, Yueliang Guo, Jia-Ming Lin and Ruey-Shiung Lin from National Taiwan University (Taipei); Li-Mei Chen from Fu-Jen Catholic University (Taipei); Pesus Chou and Song-Lih Huang from National Yang-Ming University (Taipei); Guang-Ming Shiao from Taipei Veterans General Hospital (Taipei); Kue-Hsiung Hsieh from Chang Gung University (Taoyuan); Bing-Fang Hwang, Hsien-Wen Kuo, Jim-Shoung Lai and Fung-Chang Sung from China Medical University (Taichung); Yung-Ling Lee and Huey-Jen Su from National Cheng Kung University (Tainan); Ying-Chin Ko and Ying-Chu Lin from Kaohsiung Medical University (Kaohsiung); Cheng-Kuang Shaw from Tzu-Chi University (Hualien).
}

few decades (2-8). The rising trend in prevalence was not only confined to developed countries, but also to developing countries. Some researchers, however, have pointed out the possibility that increase has slowed or ceased in children (9-13), and some have even reported prevalence as decreasing $(14,15)$. The absence of precise definitions and the lack of standardized methodologies have rendered the comparison of asthma prevalence difficult. To date, only a few articles have reported the secular trend of asthma prevalence using objective markers, such as immunoglobulin (Ig) E measurements, skin prick tests, or the measurement of bronchial hyperresponsiveness (16).

During recent years, secular trend in prevalence of childhood asthma has been examined in two Taiwanese studies. Hsieh and Shen (7) 
reported it as $1.3 \%$ in 1974 and $5.0 \%$ in 1985 among school children in Taipei City. Tsuang et al. (8) revealed the prevalence of childhood asthma as $6.5 \%$ in 1993 and $8.5 \%$ in 1997 in Tainan City. However, neither of the questionnaires used in these local studies was standardized. Till today, there has been no study that compares trends of asthma prevalence throughout Taiwan.

The standardized 'International Study of Asthma and Allergies in Childhood' (ISAAC) questionnaire has been previously validated and used throughout the world (17). It is in brief a three-phase multi-center project with phases I and III involving the administration of identical surveys to look at the time trends in childhood asthma and allergies. The use of video questionnaire has a further advantage of minimizing the linguistic problems associated with the interpretation and translation of some terms such as 'wheeze', which does not have an equivalent term in some languages (18).

To our best knowledge, there were only three ISAAC studies from Asian countries comparing secular change of asthma prevalence among school children. A previous study in Korea suggested that the prevalence rates of asthma and wheeze had increased from 1995 to 2000 (2). In Singapore, Wang et al. (10) reported a relatively small increase in asthma indices between 1994 and 2001 (10). However, in contrast to these two studies, a recent epidemiological study from Hong Kong showed that the school children aged 13-14 yr had significant decreasing prevalence in asthma from 1994 to 2002 , either in written or video questionnaires (14). Compared with data from around the world, the 12-month prevalence of asthma in Taiwan was around the lower range of the reported values (1). The aim of our study is to provide more information about the time trend of asthma in a country with a low prevalence, comparing ISAAC phase I and III data from a very large sample of school children.

\section{Subjects and methods}

Study population

Between November 1995 and March 1996, a nationwide ISAAC phase I mass screening survey was conducted for respiratory diseases and symptoms in all of the middle-school students in Taiwan. A total of 800 middle schools in Taiwan's 22 counties and more than 1 million students were investigated at that time. The study protocol has been described in detail previously $(19,20)$. Briefly, the identical standardized ISAAC-Chinese version questionnaire was taken home by students and answered by parents. Classroom incentives but not individual incentives were used to encourage participation.

Between February and June 2001, we replicated the modified ISAAC protocol and conducted phase III survey. Twenty-two middle schools were randomly chosen throughout 22 counties in Taiwan. Student sampling was stratified by grade in each school. Participants in both surveys were all between the ages of 12 and 15. Data from the 2001 survey and data from the identical 22 schools in the 1995-96 survey were combined for further analysis.

\section{Questionnaires}

The written questionnaires contained the ISAAC core questions on symptoms of asthma and allergies, and were scanned using an optical mark recognition software. All questions were translated into Chinese and back-translated into English according to the instructions of the protocol. 'Physician-diagnosed asthma' was defined by parental reports, 'Has your index child ever been diagnosed asthmatic by a physician in the child's lifetime?' 'Wheeze ever' was asked as, 'Has your index child ever had wheeze or whistling in the chest at any time in the past when he/she did not have a cold or the flu?' Information on lifetime prevalence of asthma was obtained using these definitions. 'Current' symptoms referred to symptoms occurring in the past 12 months. Severity of asthma symptoms was assessed among children with current wheeze from responses to questions about the number of wheeze attacks in the past 12 months, and about sleep disturbance owing to wheeze in the past 12 months (17).

As recommended by the ISAAC protocol, the video questionnaires were also administered to all school children. The video questionnaires showed five scenes related to asthma symptoms and severity: (i) wheeze at rest; (ii) wheeze and shortness of breath with exercise; (iii) night wheeze; (iv) night cough; and (v) severe wheeze and shortness of breath. For the two surveys, the International version ISAAC video questionnaire was used, which displayed young persons of different races. All children answered specific questions after viewing each scene (17). The study protocol was approved by the Institutional Review Board at our university hospital and it complied with the principles outlined in the Helsinki Declaration (21). 


\section{Lee et al.}

Statistical analysis

All analyses were stratified by sex. Missing or inconsistent responses were included in the denominator for prevalence calculations while they were excluded from subsequent bivariate analysis according to ISAAC recommendations $(3,9)$. Chi-square $\left(\chi^{2}\right)$ tests were used to examine for statistical significance in comparisons of proportions between the two surveys, and prevalence odds ratios (POR) and 95\% confidence intervals $(\mathrm{CI})$ were calculated. To test whether the difference between POR for time trends of different severity levels was significant, a test of homogeneity of the OR was also calculated. Statistical significance was all set at $\mathrm{p}<0.05$.

\section{Results}

In 2001, a total of 11,738 students and their parents from 22 middle schools were investigated. The response rate was $94.1 \%$. There were 44,104 data (response rate 89.3\%) selected from the identical schools in the 1995-96 survey. In both surveys, the response rates were independent and mutually exclusive of the reported diseases or symptoms. Compared with the 1995-96 survey, children in the 2001 survey seemed to be a little older (13.6 yr vs. $13.3 \mathrm{yr}$ ) and had a higher proportion of females $(50.8 \%$ vs. $49.6 \%)$. Table 1 shows the age and sex
Table 1. Demographic data of the recruited children in phase I and III surveys

\begin{tabular}{lcc}
\hline & Phase I (1995-96) & Phase III (2001) \\
\hline $\mathrm{n}$ & 44,104 & 11,048 \\
Sex & & \\
$\quad$ Boys & $22,232(50.4)$ & $5433(49.2)$ \\
$\quad$ Girls & $21,872(49.6)$ & $5615(50.8)$ \\
Age (yr) & & \\
12 & $10,733(24.3)$ & $1310(11.9)$ \\
13 & $14,939(33.9)$ & $3726(33.7)$ \\
14 & $15,214(34.5)$ & $3631(32.9)$ \\
$\quad 15$ & $3218(7.3)$ & $381(21.6)$ \\
Response rate (\%) & 89.3 & 94.1 \\
\hline
\end{tabular}

Results are shown as $\mathrm{n}(\%)$.

Percentages do not total 100 because of round up of values.

distribution of the participating children in phase I and phase III studies.

In Table 2, prevalence and POR of different asthma symptoms and severity indices from the written questionnaire are depicted. The overall prevalence of physician-diagnosed asthma significantly increased from $4.5 \%$ in phase I to $6.0 \%$ in phase III (POR 1.4, 95\% CI 1.2-1.5). The lifetime prevalence of wheeze (wheeze ever) also significantly increased from the 1995-96 survey to the 2001 survey (POR 1.3, 95\% CI 1.2-1.4). The increases over the 6-yr period were more marked among girls than boys. However, the 12-month prevalence of wheeze (current wheeze) significantly decreased from $5.9 \%$ in

Table 2. Comparison of prevalence (\%) of asthma from the written questionnaire in phase I and III surveys among Taiwanese school children

\begin{tabular}{|c|c|c|c|c|c|c|c|c|c|c|c|c|}
\hline & \multicolumn{4}{|c|}{ Boys } & \multicolumn{4}{|c|}{ Girls } & \multicolumn{4}{|c|}{ All } \\
\hline & Phase I & Phase III & POR§ & $95 \% \mathrm{Cl}$ & Phase I & Phase III & POR§ & $95 \% \mathrm{Cl}$ & Phase I & Phase III & POR\# & $95 \% \mathrm{Cl}$ \\
\hline \multicolumn{13}{|l|}{ Lifetime prevalence } \\
\hline Physician-diagnosed asthma & 5.5 & 7.0 & $1.3^{* * *}$ & $1.2-1.5$ & 3.5 & 5.0 & $1.4^{* * *}$ & $1.3-1.7$ & 4.5 & 6.0 & $1.4^{* * *}$ & $1.2-1.5$ \\
\hline Wheeze & 9.9 & 11.3 & $1.2^{* * *}$ & $1.1-1.3$ & 7.1 & 9.4 & $1.4^{* * *}$ & $1.3-1.6$ & 8.5 & 10.3 & $1.3^{* * *}$ & $1.2-1.4$ \\
\hline \multicolumn{13}{|l|}{ Prevalence of current symptoms } \\
\hline Wheeze & 5.9 & 4.1 & $0.7^{* * *}$ & $0.6-0.8$ & 4.2 & 4.0 & 1.0 & $0.9-1.2$ & 5.0 & 4.0 & $0.8^{* * *}$ & $0.7-0.9$ \\
\hline \multicolumn{13}{|l|}{ Number of wheeze attacks } \\
\hline $1-3$ & 3.9 & 2.7 & $0.7^{* * *}$ & $0.6-0.8$ & 2.8 & 2.9 & $1.1 \dagger$ & $0.9-1.3$ & 3.3 & 2.8 & $0.8^{*} \dagger$ & $0.7-1.0$ \\
\hline $4-12$ & 1.4 & 0.9 & $0.7^{*}$ & $0.5-1.0$ & 0.9 & 0.6 & $0.7^{*} \dagger$ & $0.5-1.0$ & 1.2 & 0.8 & $0.7^{*} \dagger$ & $0.6-0.9$ \\
\hline$\geq 13$ & 0.6 & 0.3 & $0.5^{* *}$ & $0.3-0.9$ & 0.4 & 0.2 & $0.7 \dagger$ & $0.4-1.2$ & 0.5 & 0.3 & $0.6^{* *} \dagger$ & $0.4-0.8$ \\
\hline \multicolumn{13}{|l|}{ Sleep disturbance due to wheeze } \\
\hline$\leq 1$ night/week & 1.9 & 1.8 & 1.0 & $0.8-1.2$ & 1.5 & 1.4 & 1.0 & $0.8-1.3$ & 1.7 & 1.6 & 1.0 & $0.8-1.2$ \\
\hline$>1$ night/week & 0.4 & 0.3 & 0.7 & $0.4-1.3$ & 0.2 & 0.2 & 0.7 & $0.4-1.4$ & 0.3 & 0.2 & 0.7 & $0.5-1.1$ \\
\hline Speech-limiting wheeze & 0.9 & 0.6 & 0.7 & $0.5-1.0$ & 0.8 & 0.8 & 1.0 & $0.7-1.5$ & 0.9 & 0.7 & 0.9 & $0.7-1.1$ \\
\hline Exercise-induced wheeze & 7.8 & 5.6 & $0.7^{* * *}$ & $0.6-0.8$ & 7.2 & 7.2 & 1.0 & $0.9-1.1$ & 7.5 & 6.4 & $0.9^{* * *}$ & $0.8-0.9$ \\
\hline Night cough & 11.9 & 10.9 & 0.9 & $0.8-1.0$ & 11.5 & 10.5 & 0.9 & $0.8-1.0$ & 11.7 & 10.7 & $0.9^{* *}$ & $0.9-1.0$ \\
\hline
\end{tabular}

${ }^{*} p<0.05$

${ }^{* *} p<0.01$.

${ }^{* * *} p<0.001$

$\dagger p<0.05$ for test of homogeneity of the odds ratios.

$\S$ Adjusted for age, and using prevalence in phase I as reference.

\#Adjusted for sex and age, and using prevalence in phase I as reference.

$\mathrm{POR}$, prevalence odds ratio; $\mathrm{Cl}$, confidence interval. 
phase I to $4.1 \%$ in phase III (POR 0.7, 95\% CI $0.6-0.8$ ) among boys, and stabilized from $4.2 \%$ in phase I to $4.0 \%$ in phase III (POR 1.0, 95\% CI 0.9-1.2) among girls. All other current asthma symptoms obtained from the written questionnaire were also fewer in phase III compared with phase I among boys, but were relatively stable between two surveys among girls (Table 2).

The amount of increase or decrease in asthma prevalence varied in both sexes. The age-adjusted POR of physician-diagnosed asthma in boys compared with girls in phase I was $1.6(95 \%$ CI $1.5-1.8)$ while in phase III it was $1.4(95 \% \mathrm{CI}$ $1.2-1.7)$. The corresponding figures for lifetime wheeze were $1.4(95 \%$ CI $1.3-1.5)$ and $1.2(95 \%$ CI 1.1-1.4), respectively. While current wheeze was considered, boys had a higher prevalence than girls in phase I (POR 1.4, 95\% CI 1.3-1.6), but there was no substantial difference between boys and girls in phase III (POR 1.0, 95\% CI $0.9-1.2)$. The relative prevalence changes between two surveys from the written questionnaire are shown in Fig. 1a. This figure illustrates that with the exception of 'night cough', girls had a higher increase in lifetime prevalence and a lower decrease in prevalence of current symptoms of asthma compared with boys between phase I and III surveys.

In Table 3, the lifetime prevalence of asthma symptoms from the five scenes in video questionnaire are presented. All of them increased significantly over the 6-yr period in both sexes, especially among girls (Fig. 1b). The 12-month prevalence of 'wheeze at rest', 'wheeze and shortness of breath with exercise' and 'night cough' also rose significantly among girls, but did not obviously change among boys (Table 3 ). Similarly, prevalence of the current symptoms from scene (iii) and (v) decreased from phase I to phase III among boys. A significantly lower proportion of boys answered positively to the scene of 'night wheeze' and 'severe wheeze and shortness of breath' in the past 12 months in 2001 than in 1995-96, with POR 0.7 (95\% CI $0.5-0.9)$ and 0.7 (95\% CI 0.6-0.9), respectively. However, these two prevalences did not alter significantly among girls (Table 3 ). Fig. 1c summarizes the relative prevalence changes of current symptoms between two surveys from the video questionnaire.

In questions of 'number of wheeze attacks' and 'number of sleep disturbances owing to wheeze per week' from the written questionnaire, the 12month prevalence between two surveys mostly decreased and were more marked on severe symptoms compared with mild symptoms in both
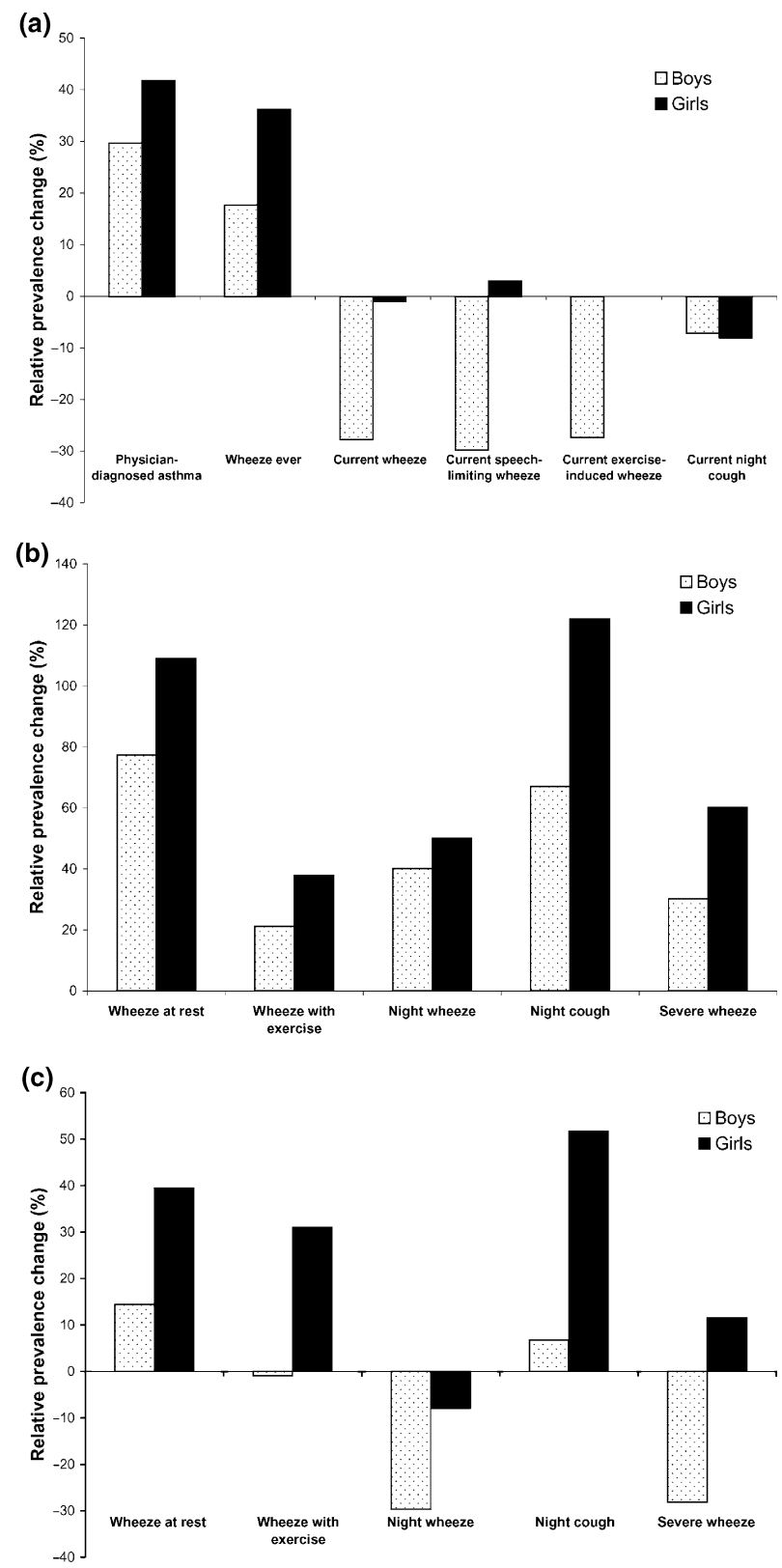

Fig. 1. Relative prevalence change in prevalences of asthma in boys and girls. (a) Written questionnaire by parent; (b) lifetime symptoms in video questionnaire by child; (c) current symptoms in video questionnaire by child. Relative prevalence change is calculated as $\{$ (phase III/phase I) -1$\} \times 100 \%$ after standardized for age.

sexes (Table 2, Fig. 2). The test of homogeneity of POR was statistically significant in subgroups of 'number of wheeze attacks' among girls, indicating a greater decrease in the higher severity category. Subgroups of 'number of wheeze attacks' among boys and subgroups of 'number of sleep disturbances owing to wheeze per week' in both sexes also showed greater decreases in prevalence but did not reach statistical significance. 


\section{Lee et al.}

Table 3. Comparison of prevalence (\%) of asthma from the video questionnaire in phase I and III surveys among Taiwanese school children

\begin{tabular}{|c|c|c|c|c|c|c|c|c|c|c|c|c|}
\hline & \multicolumn{4}{|c|}{ Boys } & \multicolumn{4}{|c|}{ Girls } & \multicolumn{4}{|c|}{ All } \\
\hline & Phase I & Phase III & POR§ & $95 \% \mathrm{Cl}$ & Phase I & Phase III & POR§ & $95 \% \mathrm{Cl}$ & Phase I & Phase III & POR\# & $95 \% \mathrm{Cl}$ \\
\hline \multicolumn{13}{|l|}{ Lifetime symptoms } \\
\hline Scene (i): wheeze at rest & 5.7 & 9.8 & $1.9^{* * *}$ & $1.7-2.1$ & 4.3 & 8.9 & $2.2^{* * *}$ & $2.0-2.5$ & 5.0 & 9.3 & $2.0^{* * *}$ & $1.9-2.2$ \\
\hline Scene (ii): wheeze with exercise & 12.4 & 14.4 & $1.3^{* * *}$ & $1.1-1.4$ & 10.0 & 13.8 & $1.4^{* * *}$ & $1.3-1.6$ & 11.2 & 14.1 & $1.3^{* * *}$ & $1.3-1.4$ \\
\hline Scene (iii): night wheeze & 3.3 & 4.5 & $1.4^{* * *}$ & $1.2-1.7$ & 2.4 & 3.5 & $1.5^{* * *}$ & $1.3-1.8$ & 2.8 & 4.0 & $1.5^{* * *}$ & $1.3-1.6$ \\
\hline Scene (iv): night cough & 4.9 & 8.1 & $1.7^{* * *}$ & $1.5-1.9$ & 4.4 & 9.8 & $2.4^{* * *}$ & $2.1-2.6$ & 4.6 & 9.0 & $2.0^{* * *}$ & $1.9-2.2$ \\
\hline Scene (v): severe wheeze & 6.4 & 8.2 & $1.3^{* * *}$ & $1.2-1.5$ & 4.7 & 7.4 & $1.7^{* * *}$ & $1.5-1.9$ & 5.6 & 7.8 & $1.5^{* * *}$ & $1.4-1.6$ \\
\hline \multicolumn{13}{|l|}{ Current symptoms } \\
\hline Scene (i): wheeze at rest & 3.5 & 3.8 & 1.2 & $1.0-1.4$ & 2.8 & 3.7 & $1.4^{* * *}$ & $1.2-1.7$ & 3.1 & 3.8 & $1.3^{* * *}$ & $1.1-1.4$ \\
\hline Scene (ii): wheeze with exercise & 8.6 & 8.0 & 1.0 & $0.9-1.1$ & 6.8 & 8.7 & $1.3^{* * *}$ & $1.2-1.5$ & 7.7 & 8.3 & $1.1^{* * *}$ & $1.1-1.2$ \\
\hline Scene (iii): night wheeze & 1.7 & 1.2 & $0.7^{* *}$ & $0.5-0.9$ & 1.3 & 1.2 & 0.9 & $0.7-1.2$ & 1.5 & 1.2 & $0.8^{*}$ & $0.7-1.0$ \\
\hline Scene (iv): night cough & 3.0 & 3.2 & 1.1 & $0.9-1.3$ & 2.9 & 4.3 & $1.5^{* * *}$ & $1.3-1.8$ & 2.9 & 3.8 & $1.3^{* * *}$ & $1.2-1.5$ \\
\hline Scene (v): severe wheeze & 3.9 & 2.7 & $0.7^{* * *}$ & $0.6-0.9$ & 3.1 & 3.3 & 1.1 & $0.9-1.3$ & 3.5 & 3.0 & 0.9 & $0.8-1.0$ \\
\hline
\end{tabular}

${ }^{*} p<0.05$.

$*^{* *} p<0.01$.

$* * * p<0.001$

$\S$ Adjusted for age, and using prevalence in phase I as reference.

\#Adjusted for sex and age, and using prevalence in phase I as reference.

$\mathrm{POR}$, prevalence odds ratio; $\mathrm{Cl}$, confidence interval.

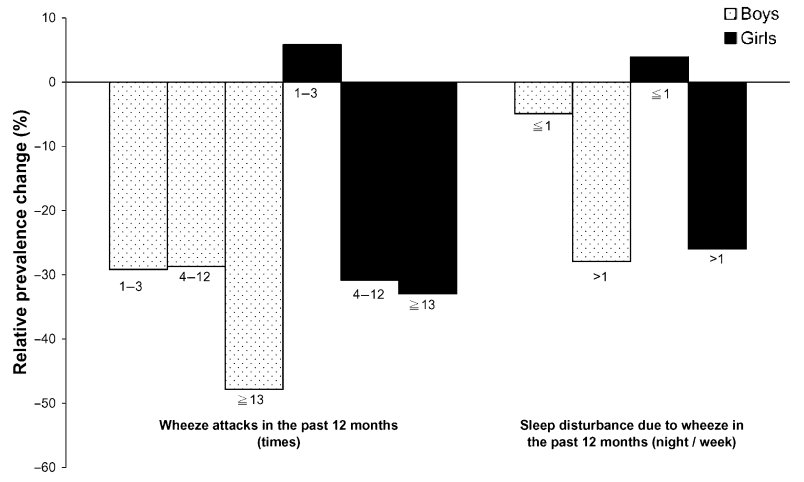

Fig. 2. Relative change in prevalence of indices of asthma severity in boys and girls. Relative prevalence change is calculated as $\{($ phase III/phase I $)-1\} \times 100 \%$ after standardized for age.

\section{Discussion}

With the rigorous application of identical study methods and the use of a large representative sample within the general population, we believe that our national data is credible for time trends of the prevalence of asthma indices among Taiwanese school children. Using ISAAC core written and video questionnaires, we found that there existed a general tendency toward an increase in lifetime prevalence of physician-diagnosed asthma and asthma symptoms over the 6-yr period, more marked for girls than for boys. Most of the current symptoms of asthma decreased among boys but stabilized among girls. When comparing different severity levels, we noted that the decreasing trends of current symptoms were more marked on severe symptoms compared with mild symptoms in both sexes.

Over the past decades, many epidemiological studies in different parts of the world have shown an apparent changing trend of asthma prevalence. Most of these studies were carried out using non-standardized written questionnaires without validation. It is conceivable that some of the documented change by previous reports such as those from Taiwan might be because of methodological differences $(7,8)$. In order to minimize technical bias, we analyzed data from the same schools and used standardized procedures for data collection. As the identical ISAAC questionnaires were applied to school children of similar ages, it is most unlikely that the differences in our findings are attributable to any changes of methodology. However, owing to the lack of objective data, the trend in our repeated cross-sectional studies should be interpreted with caution $(6,16)$.

In our results, significant increases were noted in lifetime prevalence of physician-diagnosed asthma and wheeze in Taiwanese school children from 1995 to 2001. The comparison from our two surveys showed a 1.3- to 1.4-fold increase in the lifetime prevalence (Table 2), suggesting an approximately $5 \%$ elevation per year on average. Similar increases in prevalence have been reported all around the world $(6,11,22)$. In children with a physician's diagnosis of asthma, $77.3 \%$ in phase I and $80.2 \%$ in phase III were reported as ever having wheeze while in those without such a diagnosis, only $5.2 \%$ and $5.9 \%$ reported such 
symptoms (both $\mathrm{p}<0.001$ ). Of 3734 children who had ever wheezed in phase I survey, a diagnosis of asthma was known in 1547 instances $(41.4 \%)$. In contrast, a diagnosis of asthma was known in $46.6 \%$ of the children who had ever wheezed in phase III survey. This does not necessarily mean the increase is attributable to increased recognition and awareness among the general population and medical personnel. The adjusted POR of non-asthmatic wheeze was 1.2 (95\% CI 1.0-1.3) between two surveys. Although the trends may be due in part to changes in the reporting and consulting behavior of parents and in the labeling of the disease by general practitioners $(6,23)$, it does mean that some of these increases are real. As the prevalence of nonasthmatic wheeze still increased, the rise in lifetime prevalence of asthma could not be attributed solely to a change in diagnostic terminology or public awareness.

In 1995-96, the prevalence of current wheeze in Taiwan was low in comparison with international data (1). It was anticipated that it would increase with rising affluence and urbanization (24). Unexpectedly, we did not observe a consistent increase in prevalence of current symptoms. In fact, there was a decrease in most of the indices (Table 2). This result is in keeping with some recent studies, which suggest that the asthma burden may be plateauing (11). In Taiwan, the obligatory National Health Insurance was administered since 1995 and more than $99 \%$ of Taiwanese residents were covered. Although not fully understood, improvement in therapeutic management, compliance, accessibility of primary care or prevention efforts over time might in part explain the declining current symptoms (15). Another possible reason is that the environmental factors that have resulted in this epidemic have 'recruited' all genetically susceptible individuals in these years (12).

In questions of severity indices from the written questionnaire, we also found that the 12-month prevalence between the two surveys were more marked on severe symptoms compared with mild symptoms (Table 2, Fig. 2), which was consistent with the results from the video questionnaire. In the video questionnaire, because scenes (iii) (night wheeze) and (v) (severe wheeze and shortness of breath) showed a relatively severe case of asthma, the POR of these two symptoms in the past 12 months were lower than those of the other three scenes in both sexes (Table 3). A recent ISAAC survey among school children in England suggested that the increases in 12-month symptoms of asthma were confined to mild symptoms (5), arguing that improved therapy could have affected the severity profile of asthma symptoms, which would lead to an increase of less severe symptoms (5, 25). Although details of asthma therapy on subjects in both surveys were not available, indirect evidence suggested that asthma care among the patients had changed. It is reasonable to speculate that the decrease in the severity of symptoms among the asthma subjects documented in this study might have been partly because of improved asthma care.

Apart from translation and interpretation problems, the ISAAC video questionnaire has been suggested to be more objective than the written one (26). As for our video questionnaire results, all lifetime prevalence of asthma significantly increased in both sexes (Table 3). Except for some scenes in which the prevalence of current symptoms still rose among girls, most of the responses to the written and video questionnaires draw an analogous picture of the trends toward an increase in lifetime prevalence of asthma, more marked among girls, and a decrease in current asthma symptoms and severity, mostly among boys, which strongly corroborated the observed trends.

Sex difference in time trends was another important finding in our study, which might be overlooked without gender stratification $(5,11)$. Generally, girls had a higher increase in lifetime prevalence and a lower decrease in prevalence of current symptoms of asthma compared with boys (Table 2, Fig. 1a). In England, Venn et al. (27) looked at time trends of asthma between 1988 and 1995 among school children aged 4-11 yr and found that the increase in asthma symptoms was greater in girls than in boys. Maziak et al. (3) performed two secular ISAAC surveys among 13- to 14-year-old school children in Germany and also found that girls had a higher increase of asthma than boys. Similar sex-related trends in the occurrence of asthma were also reported among 7- to 13-year-old school children in Norway (28). Speculating on the reasons behind the observed female-led tendency is not easy. The natural history of asthma points toward male predominance in childhood and a female predominance from adolescence $(29,30)$. Considering this natural history, our results showed that the noticed sex-related trends would alleviate the sex difference in prevalence of childhood asthma, and would probably augment it toward more female dominance in future years.

Regardless of time trends, in most of the questions from both surveys, we also noted that girls were less likely to report asthma in video questionnaire and to be reported as having 
asthma in written questionnaire. Our data suggested that the discrepancy in prevalence was most likely to reflect underdiagnosis and underreport of asthma among girls, which was in accordance with the conclusions from previous studies among Swiss and Danish school children $(31,32)$. Some environmental influences associated with more traditional lifestyles can confer resistance to the development of allergies even in susceptible individuals. It is plausible that because girls are relatively inactive and spend more time at home, lifestyles become more critical and interfere with children's ability to develop normal resistance to asthma (33).

The seasons of investigation in phase I and phase III surveys were not identical in our study. However, because the main outcome measurements were lifetime and 12-month prevalence of asthma, season of investigation would not be a major confounder in our design. We also believed that the calculated estimates could reflect to a good extent the morbidity of asthma in each survey. In fact, in the previous analysis from our population, season of investigation did not show significant differences in association with asthma (19).

Many personal and environmental factors contribute to asthma attacks and their prevalence in childhood $(12,20,34)$. There were also possibly changes over time in the proportions of these factors between asthmatics, which has created considerable concern about what kinds of factors might be responsible for the time trends. To date, however, population studies exploring the effects of these variables remained limited. We believed that changes in genetic susceptibility to wheeze, as a response of atopy, were unlikely to have occurred between 1995-96 and 2001. It has also been hypothesized that environmental rather than genetic causes were responsible for the change of asthma prevalence (35). In our previous analysis, no specific factors were considered significant in explaining the observed changes in asthma prevalence (19). However, we accidentally found that the increasing temperature during these years might be an important reason for the rising trends of the lifetime prevalence of asthma (19). Future studies are required to investigate the contribution of changes over time in other potential etiological factors, including socio-economic status, family size, early childhood infections and hygiene, pollutant and allergen exposure, diet and obesity, and perinatal risk factors.

In conclusion, we used the same standardized and validated ISAAC questionnaires to study the prevalence of asthma in Taiwanese school children between 1995-96 and 2001. The present study suggested a general trend toward an increase in lifetime prevalence of asthma indices, mostly among girls, but a decrease in current asthma symptoms, mostly among boys. The decreasing trends of current symptoms were more marked on severe symptoms compared with mild symptoms. A combination of both improved awareness and management of asthma might in part explain the whole circumstance. Further work, including objective measurements, is necessary not only to clarify the real trends of asthma prevalence in the new millennium, but to also identify the kinds of factors that might be responsible for these changes.

\section{Acknowledgments}

This study was partially supported by grant \#NSC87-2621P-006-013 from National Science Council and grant \# DOH90-TD-1138 from Department of Health, and partially funded by Environmental Protection Administration in Taiwan.

\section{References}

1. The International Study of Asthma and Allergies in Childhood Steering Committee. Worldwide variation in prevalence of symptoms of asthma, allergic rhinoconjuntivitis, and atopic eczema: ISAAC. Lancet 1998: 351: 1225-32.

2. Hong SJ, LeE MS, SoHn MH, et al. Self-reported prevalence and risk factors of asthma among Korean adolescents: 5-year follow-up study, 1995-2000. Clin Exp Allergy 2004: 34: 1556-62.

3. Maziak W, Behrens T, Brasky TM, et al. Are asthma and allergies in children and adolescents increasing? Results from ISAAC phase I and phase III surveys in Munster, Germany. Allergy 2003: 58: 572-9.

4. Heinrich J, Hoelscher B, Frye C, Meyer I, Wust M, Wichmann HE. Trends in prevalence of atopic diseases and allergic sensitization in children in Eastern Germany. Eur Respir J 2002: 19: 1040-6.

5. Ng Man Kwong G, Proctor A, Billings C, et al. Increasing prevalence of asthma diagnosis and symptoms in children is confined to mild symptoms. Thorax 2001: 56: 312-4.

6. Magnus P, JAAKKola JJ. Secular trend in the occurrence of asthma among children and young adults: critical appraisal of repeated cross sectional surveys. BMJ 1997: 314: 1795-9.

7. Hsien KH, SHen JJ. Prevalence of childhood asthma in Taipei, Taiwan and other Asian Pacific countries. J Asthma 1988: 25: 73-82.

8. Tsuang HC, Su HJ, Kao FF, Shin HC. Effects of changing risk factors on increasing asthma prevalence in southern Taiwan. Paediatr Perinat Epidemiol 2003: 17: 3-9.

9. Garcia-Marcos L, Quiros AB, Hernandez GG, et al. Stabilization of asthma prevalence among adolescents and increase among schoolchildren (ISAAC phases I and III) in Spain. Allergy 2004: 59: 1301-7. 
10. WANG XS, TAN TN, SHEK LP, et al. The prevalence of asthma and allergies in Singapore: data from two ISAAC surveys seven years apart. Arch Dis Child 2004: 89: 423-6.

11. Akinbami LJ, Schoendorf KC. Trends in childhood asthma: prevalence, health care utilization, and mortality. Pediatrics 2002: 100: 315-22.

12. Ronchetti R, Villa MP, Barreto M, et al. Is the increase in childhood asthma coming to an end? Findings from three surveys of schoolchildren in Rome, Italy. Eur Respir J 2001: 17: 881-6.

13. Kalyoncu AF, Selcuk ZT, Enunlu T, et al. Prevalence of asthma and allergic diseases in primary school children in Ankara, Turkey: two cross-sectional studies, five years apart. Pediatr Allergy Immunol 1999: 10: 261-5.

14. Wong GW, Leung TF, Ko FW, et al. Declining asthma prevalence in Hong Kong Chinese schoolchildren. Clin Exp Allergy 2004: 34: 1550-5.

15. Fleming DM, Sunderland R, Cross KW, Ross AM. Declining incidence of episodes of asthma: a study of trends in new episodes presenting to general practitioners in the period 1989-98. Thorax 2000: 55: 657-61.

16. Wieringa MH, Vermeire PA, Brunekreef B, Weyler JJ. Increased occurrence of asthma and allergy: critical appraisal of studies using allergic sensitization, bronchial hyper-responsiveness and lung function measurements. Clin Exp Allergy 2001: 31: 1553-63.

17. Asher Mi, KeIl U, Anderson HR, et al. International Study of Asthma and Allergies in Childhood (ISAAC): rationale and methods. Eur Respir J 1995: 8: 483-91.

18. Asher MI, WeIland SK. The International Study of Asthma and Allergies in Childhood (ISAAC). ISAAC Steering Committee. Clin Exp Allergy 1998: 28: 52-66.

19. LeE YL, Lin YC, Hwang BF, GuO YL. Changing prevalence of asthma in Taiwanese adolescents: two surveys 6 years apart. Pediatr Allergy Immunol 2005: 16: $157-64$.

20. Guo YL, Lin YC, Sung FC, et al. Climate, trafficrelated air pollutants and asthma prevalence in middleschool children in Taiwan. Environ Health Perspect 1999: 107: 1001-6.

21. 41st World Medical Assembly. Declaration of Helsinki: Recommendations guiding physicians in biomedical research involving human subjects. Bulletin of the Pan American Health Organization 1990: 24: 606-9.

22. Lewis S, Butland B, Strachan D, et al. Study of the aetiology of wheezing illness at age 16 in two national British birth cohorts. Thorax 1996: 51: 670-6.

23. Barraclough R, Devereux G, Hendrick DJ, StenTON SC. Apparent but not real increase in asthma prevalence during the 1990s. Eur Respir J 2002: 20: 826-33.

24. Aligne CA, Auinger P, Byrd RS, Weitzman M. Risk factors for pediatric asthma. Contributions of poverty, race, and urban residence. Am J Respir Crit Care Med 2000: 162: 873-7.

25. Anderson HR, Butland BK, Strachan DP. Trends in prevalence and severity of childhood asthma. BMJ 1994: 308: 1600-4.

26. Lai CK, Chan JK, Chan A, et al. Comparison of the ISAAC video questionnaire (AVQ3.0) with the ISAAC written questionnaire for estimating asthma associated with bronchial hyperreactivity. Clin Exp Allergy 1997: 27: $540-5$.

27. Venn A, Lewis S, Cooper M, Hill J, Britton J. Increasing prevalence of wheeze and asthma in Nottingham primary schoolchildren 1988-1995. Eur Respir J 1998: 11: 1324-8.

28. Selnes A, Bolle R, Holt J, Lund E. Cumulative incidence of asthma and allergy in north-Norwegian schoolchildren in 1985 and 1995. Pediatr Allergy Immunol 2002: 13: 58-63.

29. Strachan DP. Epidemiology of rhinitis. In: BuSSE WW, Holgate ST, eds. Asthma and Rhinitis, 2nd edn. Oxford: Blackwell Scientific Publications 2000: 33-42.

30. Stender M, Weiland SK, Duhme H, Keil U. Epidemiology of asthma and allergic disease. In: Environmental Health Criteria 212, Principles and Methods for Assessing Allergic Hypersensitization Associated with Exposure to Chemicals. Geneva: World Health Organization, 1999: 224-71.

31. Sennhauser FH, Kunni CE. Prevalence of respiratory symptoms in Swiss children: is bronchial asthma really more prevalent in boys? Pediatr Pulmonol 1995: 19: 161-6.

32. Siersted hC, Boldsen J, Hansen hS, Mostgaard G, Hyldebrandt N. Population based study of risk factors for underdiagnosis of asthma in adolescence: Odense schoolchild study. BMJ 1998: 316: 651-5.

33. MAZIAK W. Asthma and the exposure-disease tenet. J Clin Epidemiol 2002: 55: 737-40.

34. Lee YL, Lin YC, Hsiue TR, Hwang BF, Guo YL. Indoor/outdoor environmental exposures, parental atopy, and physician-diagnosed asthma in Taiwanese schoolchildren. Pediatrics 2003: 112: e389-95.

35. D'Amato G, Liccardi G, D’Amato M, Cazzola M. Outdoor air pollution, climatic changes and allergic bronchial asthma. Eur Respir J 2002: 20: $763-$ 76 . 
This document is a scanned copy of a printed document. No warranty is given about the accuracy of the copy. Users should refer to the original published version of the material. 